\title{
Probing the high- $x$ content of the nuclei in the fixed-target mode at the LHC
}

A. Kusina ${ }^{* a}$, C. Hadjidakis ${ }^{b}$, D. Kikoła ${ }^{c}$, J.P. Lansberg ${ }^{b}$, L. Massacrier ${ }^{b}$, M.G. Echevarria ${ }^{d}$, I. Schienbein ${ }^{e}$, J. Seixas ${ }^{f, g}$, H.S. Shao ${ }^{h}$, A. Signori ${ }^{i}$, B. Trzeciak $^{j}$, S.J. Brodsky ${ }^{k}$, G. Cavoto ${ }^{l}$, C. Da Silva ${ }^{m}$, F. Donato ${ }^{n}$, E.G. Ferreiro ${ }^{o, p}$, I. Hřivnáčová ${ }^{b}$, A. Klein ${ }^{m}$, A. Kurepin ${ }^{r}$, C. Lorcé ${ }^{s}$, F. Lyonnet ${ }^{t}$, Y. Makdisi ${ }^{u}$, S. Porteboeuf ${ }^{w}$, C. Quintans ${ }^{g}$, A. Rakotozafindrabe ${ }^{x}$, P. Robbe ${ }^{y}$, W. Scandale ${ }^{z}$, N. Topilskaya ${ }^{r}$, A. Uras ${ }^{a a}$, J. Wagner ${ }^{a b}$, N. Yamanaka ${ }^{b}$, Z. Yang ${ }^{a c}$, A. Zelenski ${ }^{u}$

${ }^{a}$ Institute of Nuclear Physics Polish Academy of Sciences, PL-31342 Krakow, Poland

${ }^{b}$ IPNO, CNRS-IN2P3, Univ. Paris-Sud, Université Paris-Saclay, 91406 Orsay Cedex, France

${ }^{c}$ Faculty of Physics, Warsaw University of Technology, ul. Koszykowa 75, 00-662 Warsaw, Poland

${ }^{d}$ Istituto Nazionale di Fisica Nucleare, Sezione di Pavia, via Bassi 6, 27100 Pavia, Italy

${ }^{e}$ Laboratoire de Physique Subatomique et de Cosmologie, Université Grenoble Alpes, CNRS/IN2P3, 53 Avenue des Martyrs, F-38026 Grenoble, France

${ }^{f}$ Dep. Fisica, Instituto Superior Tecnico, Av. Rovisco Pais 1, 1049-001 Lisboa, Portugal

${ }^{g}$ LIP, Av. Prof. Gama Pinto, 2, 1649-003 Lisboa,Portugal

${ }^{h}$ LPTHE, UMR 7589, Sorbonne Universityé et CNRS, 4 place Jussieu, 75252 Paris, France

${ }^{i}$ Physics Division, Argonne National Laboratory, Lemont, IL 60439, USA

${ }^{j}$ Institute for Subatomic Physics, Utrecht University, Utrecht, The Netherlands

${ }^{k}$ SLAC National Accelerator Laboratory, Stanford University, Menlo Park, CA 94025, USA

l "Sapienza" Università di Roma, Dipartimento di Fisica \& INFN, Sez. di Roma, P.le A. Moro 2, 00185 Roma, Italy

${ }^{m}$ P-25, Los Alamos National Laboratory, Los Alamos, NM 87545, USA

${ }^{n}$ Turin University, Department of Physics, and INFN, Sezione of Turin, Turin, Italy

${ }^{o}$ Dept. de Física de Partículas \& IGFAE, Universidade de Santiago de Compostela, 15782 Santiago de Compostela, Spain

${ }^{p}$ Laboratoire Leprince-Ringuet, Ecole polytechnique, CNRS/IN2P3, Université Paris-Saclay, Palaiseau, France

${ }^{r}$ Institute for Nuclear Research, Moscow, Russia

${ }^{s}$ CPHT, École Polytechnique, CNRS, 91128 Palaiseau, France

${ }^{t}$ Southern Methodist University, Dallas, TX 75275, USA

"Brookhaven National Laboratory, Collider Accelerator Department

${ }^{w}$ Université Clermont Auvergne, CNRS/IN2P3, LPC, F-63000 Clermont-Ferrand, France

${ }^{x}$ IRFU/DPhN, CEA Saclay, 91191 Gif-sur-Yvette Cedex, France

${ }^{y}$ LAL, Université Paris-Sud, CNRS/IN2P3, Orsay, France

${ }^{z}$ CERN, European Organization for Nuclear Research, 1211 Geneva 23, Switzerland

${ }^{a a} I P N L$, Université Claude Bernard Lyon-I and CNRS-IN2P3, Villeurbanne, France

${ }^{a b}$ National Centre for Nuclear Research (NCBJ), Hoża 69, 00-681, Warsaw, Poland

${ }^{a c}$ Center for High Energy Physics, Department of Engineering Physics, Tsinghua University, Beijing, China

Using the LHCb and ALICE detectors in the fixed-target mode at the LHC offers unprecedented possibilities to study the quark, gluon and heavy-quark content of the proton and nuclei in the poorly known region of the high-momentum fractions. We review our projections for studies of Drell-Yan, charm, beauty and quarkonium production with both detector set-ups used with various nuclear targets and the LHC proton beams. Based on this, we show the expected improvement in the determination of the quark, charm and gluon proton and nuclear PDFs as well as discuss the implication for a better understanding of the cold-nuclear-matter effects in hard-probe production in proton-nucleus collisions.

International Conference on Hard and Electromagnetic Probes of High-Energy Nuclear Collisions 30 September - 5 October 2018

Aix-Les-Bains, Savoie, France

* Speaker. 


\section{Introduction}

In order to complement the current program of the LHC experiments, the LHC beam can be used to run in a fixed target mode. A fixed target experiment has a number of advantages that were discussed in detail within the scope of the AFTER@LHC proposal [1, 2], here we just mention the main ones: (i) access to the far backward c.m.s. region, (ii) possibility of using various targets including ${ }^{3} \mathrm{He}$, (iii) target polarisation giving access to measurements of single spin asymmetry at large momentum fractions. For the details on the design and technical implementation of the experiment we refer the reader to the full report [2] we just want to highlight here that scenarios using either a new detector or one of the existing detectors (LHCb or ALICE) were consider; in both cases allowing for running alongside the current collider program of the LHC. A three main physics subjects have been studied (i) the high fractional momentum $(x)$ frontier in nucleons and nuclei, (ii) spin content of the nucleons, and (iii) heavy-ion physics. In this contribution we concentrate on the first one - the high- $x$ frontier - for the results on the remaining topics we refer the reader to the report [2] and to other contributions from the Hard Probes 2018 conference [3, 4, 5, 6].

Scenarios using a $7 \mathrm{TeV}$ proton beam and a $2.76 \mathrm{TeV}$ lead beam were considered. In the fixed target mode these correspond to the nucleon-nucleon center of mass energy (c.m.s.) of $\sqrt{s_{N N}}=115$ $\mathrm{GeV}$ in the proton case and $\sqrt{s_{N N}}=72 \mathrm{GeV}$ in the lead case. The high-energy of the beams translates into a big pseudorapidity shift between the laboratory and c.m.s. frames which is 4.8 for the proton beam and 4.3 for the lead beam. This means that we obtain a backward detector covering $y_{\text {c.m.s. }}<0$ and negative Feynman $x\left(x_{B}\right)$. The acceptance of the LHCb and ALICE detectors in the fixed target mode is shown in Fig. 1 in comparison with the collider mode and experiments at RHIC, showing clearly the uniqueness of such a setup.

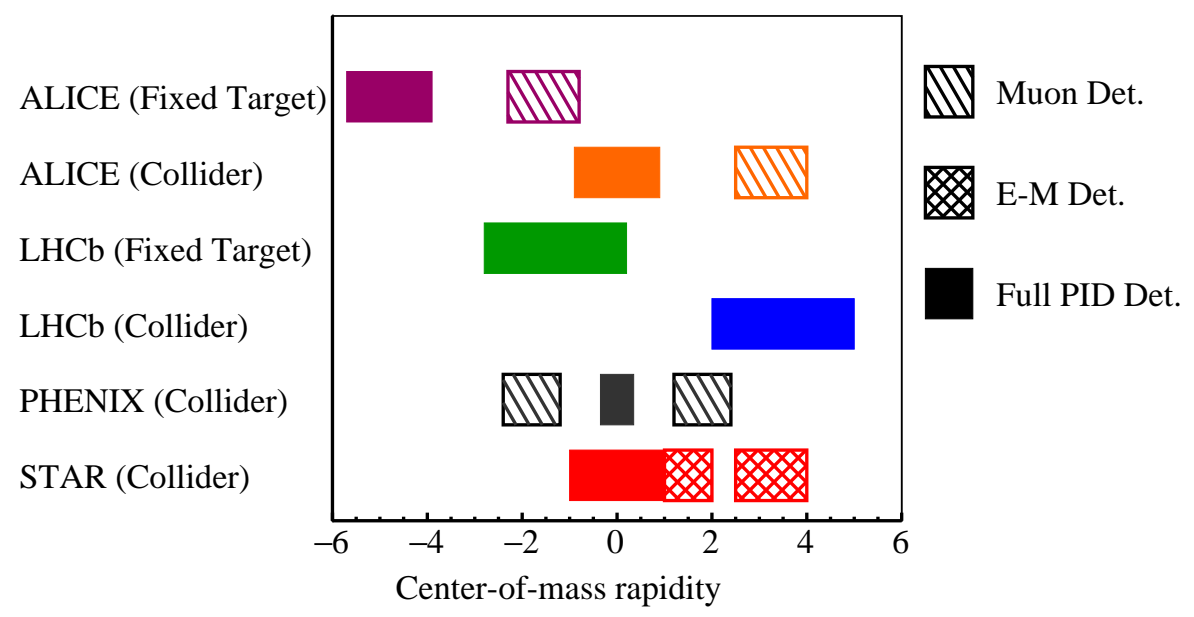

Figure 1: Comparison of the kinematic coverages of the ALICE and LHCb detectors at the LHC and the STAR and PHENIX detectors at RHIC. For ALICE and LHCb, the acceptance is shown in the collider and the fixed-target modes with a target position at the nominal Interaction Point (IP) for a $7 \mathrm{TeV}$ proton beam. The "Full PID Det." label indicates detector with particle identification capabilities, "E-M Det." - an electromagnetic calorimeter, "Muon Det." - a muon detector. Figure from [2]. 


\section{High $x$ frontier}

We review here selected projections of the impact of the fixed target experiment on proton and nuclear parton distribution functions (PDFs). The projections have been performed for the case of proton beam colliding either with protons or heavy targets in both cases leading to nucleon-nucleon c.m.s. energy of $\sqrt{s_{N N}}=115 \mathrm{GeV}$. We assume integrated luminosity of $10 \mathrm{fb}^{-1}$ in the $p p$ case and $100 \mathrm{pb}^{-1}$ in proton-nucleus case (where we considered the Xe and $\mathrm{W}$ nuclei). The projections were done using either the profiling $[7,8]$ or the reweighting method $[9,10]$.

We start with the Drell-Yan lepton pair production process. In the $p p$ case, pseudo-data for rapidity distributions in selected bins of the lepton pair invariant mass: $M_{\mu \mu} \in[4,5],[5,6],[6,7],[7,8]$ $\mathrm{GeV}$ and $M_{\mu \mu}>10.5 \mathrm{GeV}$ have been used; assuming the acceptance of the LHCb detector: $2<\eta_{\text {lab }}<5$ (giving $-2.8<y_{\text {c.m.s. }}<0.2$ ) and $p_{T}^{\mu}>1.2 \mathrm{GeV}$. The results of using these pseudo-data on the uncertainties of the CT14 PDFs [11] is presented in Figs. 2a and 2b for the case of $u$ and $d$ distributions. We can see a huge reduction of uncertainties in the high- $x$ region for the $u$ quark and a moderate reduction for the $d$ quark; a smaller decrease is also observed for the light sea quarks. Additionally, a sizable decrease of the PDF uncertainties is also found in the intermediate and small $x$ region which can be seen e.g. in Fig. 15 in [2].

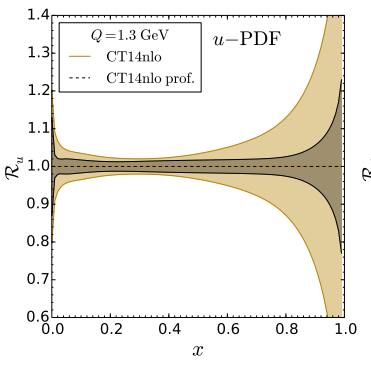

(a) $p p$ case

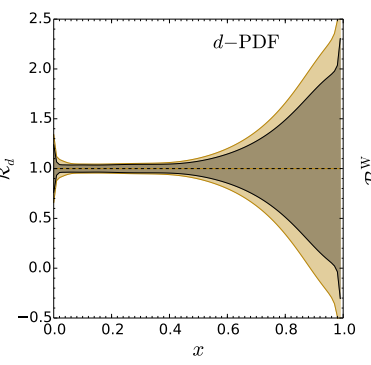

(b) $p p$ case

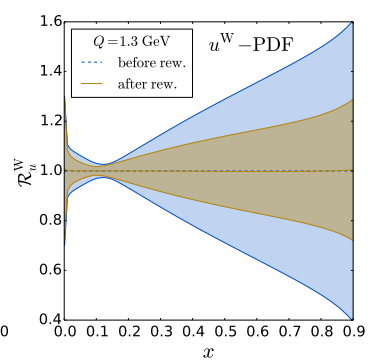

(c) $p \mathrm{~A}$ case

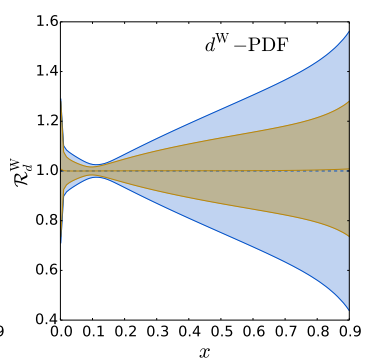

(d) $p$ A case

Figure 2: (a,b) Impact of the Drell-Yan lepton pair production in $p p$ collisions at $\sqrt{s_{N N}}=115 \mathrm{GeV}$ on the PDF uncertainties. The $u$ and $d$ PDFs from CT14 [11] are plotted as a function of $x$ at a scale $Q=1.3$ $\mathrm{GeV}$ before and after including AFTER @LHCb pseudo-data using the profiling method. (c,d) nCTEQ15 nPDFs [12] before and after the reweighting using $R_{p \mathrm{~W}}^{\mathrm{DY}}$ and $R_{p \mathrm{Xe}}^{\mathrm{DY}}$ AFTER @LHCb pseudo-data. The plots show ratio of nPDFs for tungsten (W) and the corresponding uncertainties compared to the central value at the scale $Q=1.3 \mathrm{GeV}$. Figure from [2].

In the nuclear case pseudo-data for the nuclear modifications of rapidity distributions in $p \mathrm{Xe}$ and $p \mathrm{~W}$ collisions have been used; also assuming the acceptance of the LHCb detector and using the same bins in the lepton pair invariant mass. In Figs. $2 \mathrm{c}$ and $2 \mathrm{~d}$ we present the impact of these pseudo-data on the nCTEQ15 [12] nuclear PDFs (nPDFs) in case of tungsten for $u$ and $d$ distributions. In both cases a very substantial reduction of the uncertainties is observed. We should highlight here that in reality the nPDF uncertainties are typically underestimated and in practice this reduction is even larger.

As a second example we concentrate on the nuclear gluon distribution which is one of the least known nPDFs. One example of data that can be used in order to learn about the gluon nPDF comes from heavy flavour production. The collider LHC data for heavy flavour production in both $p p$ and 
$p \mathrm{~Pb}$ collisions have been already used to constrain the small- $x$ gluon $[13,14,15]$, here we show that the corresponding data collected in the fixed target mode can also be used to study the high- $x$ gluon distribution. In Fig. 3 we present results of reweighting of the nCTEQ15 nPDFs using pseudo-data for the production of heavy flavour mesons $\left(D^{0}, J / \psi, B^{ \pm}, \Upsilon(1 S)\right)$ in $p$ Xe collisions at $\sqrt{s_{N N}}=115$ $\mathrm{GeV}$ using the HELAC-Onia framework $[16,17]$. We can see that in all cases it allows for a huge reduction of the $\mathrm{nPDF}$ uncertainties of the gluon distribution at high- $x$, even taking into account the relatively large scale uncertainties.
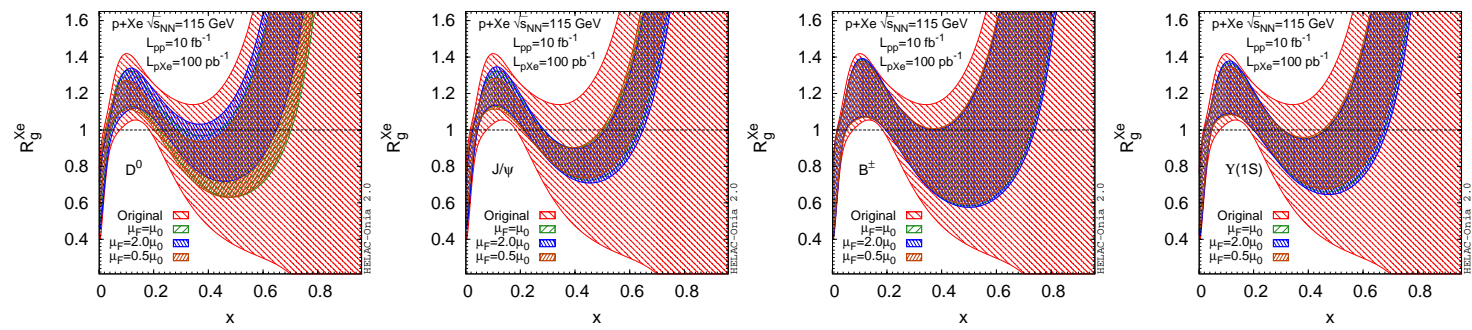

Figure 3: nCTEQ15 nPDFs before and after the reweighting using $R_{p X e}$ pseudo-data for (a) $D^{0}$, (b) $J / \psi$, (c) $B^{+}$, (d) $\Upsilon(1 S)$ production at AFTER @LHCb. The plots show ratios $R_{g}^{X e}$ of gluon densities encoded in nCTEQ15 over that in CT14 PDFs at scale $Q=2 \mathrm{GeV}$. Figure from [2].

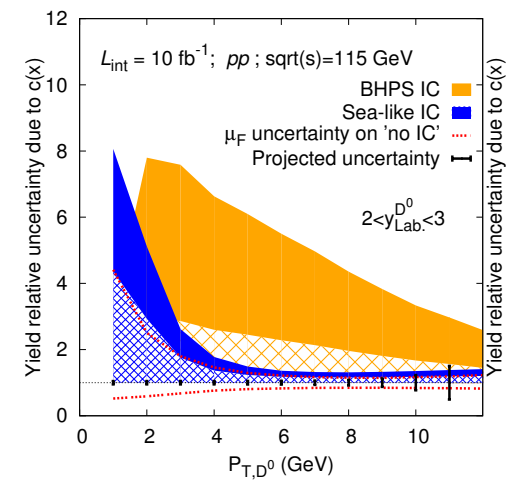

(a) $2<y_{\text {Lab }}<3$

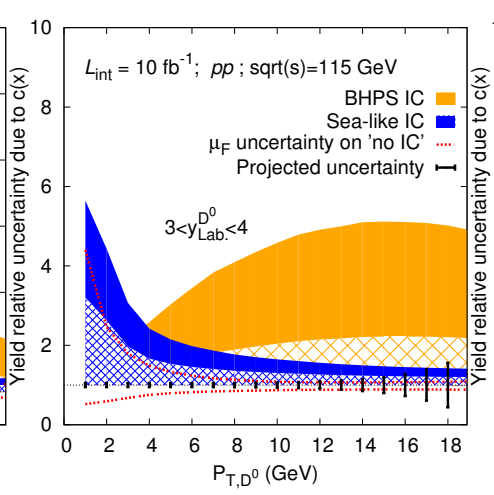

(b) $3<y_{\text {Lab }}<4$

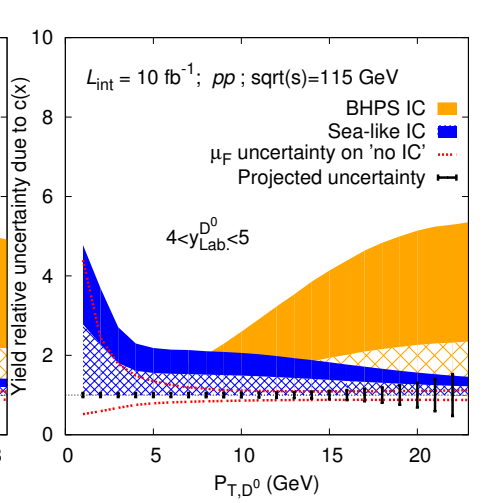

(c) $4<y_{\text {Lab }}<5$

Figure 4: Impact of the uncertainties on the charm content of the proton on the $D^{0}$ yield as a function of $p_{T}$ compared to the projected uncertainties from the measurement of the $D^{0}$ yield in $p p$ collisions at $\sqrt{s_{N N}}=115$ $\mathrm{GeV}$ in the LHCb acceptance. The orange and blue zones correspond to BHPS [19] and sea-like [20] intrinsic charm (IC) models. The filled areas correspond to yields computed with up to $\left\langle x_{\bar{c}}\right\rangle=2 \%$ (resp. 2.4\%) and the hashed areas up to $\left\langle x_{c+\bar{c}}\right\rangle=0.57 \%$ (resp. $1.1 \%$ ). The dashed red lines indicate the factorization scale uncertainty on the "no-IC" yield. Figure from [2].

The last example we present in this proceedings is the possible impact of the fixed target AFTER@LHC experiment on the heavy quark PDFs in the proton. In particular, its possibilities to confirm or strongly constrain the models of the non-perturbative (intrinsic) charm that typically predict a substantial component of the heavy quark distribution located at high- $x$ [18]. In Fig. 4 we show the relative yield uncertainty for inclusive $D^{0}$ meson production for three rapidity bins $\left(2<y_{\text {lab }}<3,3<y_{\text {lab }}<4,4<y_{\text {lab }}<5\right)$ as a function of the transverse momentum of the $D^{0}$ meson. As can be seen, even for $p_{T} \lesssim 15 \mathrm{GeV}$ the expected precision of the measurement will clearly allow to considerably constrain the intrinsic-charm model, by up to an order magnitude. 


\section{Summary}

We have presented a selection of results from [2] showing the impact of a fixed target experiment with LHC beams on our current knowledge of proton and nuclear PDFs. From these results it is clear that such an experiment would be invaluable in learning about large- $x$ distributions of quarks and gluons in both proton and nuclei. It would provide a unique opportunity for studying the high- $x$ regime which is hard to access in a collider mode of the LHC and at the same time it is important for answering many important questions like: (i) understanding the confinement properties of QCD, (ii) understanding the origin of the EMC effect, (iii) discriminating between different models of hadronic structure, (iv) testing the existence of intrinsic heavy quarks in the proton, (v) reducing the uncertainty on prompt neutrino fluxes, or (vi) improving our knowledge of parton luminosities at existing and future hadron colliders (LHC, HE-LHC, FCC-hh) which will help with searches of new heavy particles. The full results of the studies from the AFTER @LHC group can be found in [2] in particular topics related to spin physics and heavy ion collisions which were not covered here.

\section{References}

[1] S. J. Brodsky, F. Fleuret, C. Hadjidakis, and J. P. Lansberg, Phys. Rept. 522 (2013) 239, 1202 . 6585.

[2] C. Hadjidakis et al., 1807.00603.

[3] N. Yamanaka, PoS(HardProbes2018)184.

[4] C. Hadjidakis, PoS(HardProbes2018)041.

[5] A. Uras, PoS(HardProbes2018)134.

[6] J. Lansberg, PoS(HardProbes2018)019.

[7] H. Paukkunen and P. Zurita, JHEP 12 (2014) 100, 1402 . 6623.

[8] S. Alekhin et al., Eur. Phys. J. C75 (2015), no. 7 304, 1410.4412.

[9] W. T. Giele and S. Keller, Phys. Rev. D58 (1998) 094023, hep-ph / 9803393.

[10] A. Kusina, et al., Eur. Phys. J. C77 (2017), no. 7 488, 1610.02925.

[11] S. Dulat, et al., Phys. Rev. D93 (2016), no. 3 033006, 1506.07443.

[12] K. Kovarik et al., Phys. Rev. D93 (2016), no. 8 085037, 1509.00792.

[13] PROSA Collaboration, O. Zenaiev et al., Eur. Phys. J. C75 (2015), no. 8 396, 1503.04581.

[14] R. Gauld and J. Rojo, Phys. Rev. Lett. 118 (2017), no. 7 072001, 1610.09373.

[15] A. Kusina, J.-P. Lansberg, I. Schienbein, and H.-S. Shao, Phys. Rev. Lett. 121 (2018) 052004, 1712.07024.

[16] H.-S. Shao, Comput. Phys. Commun. 184 (2013) 2562-2570, 1212.5293.

[17] H.-S. Shao, Comput. Phys. Commun. 198 (2016) 238-259, 1507.03435.

[18] S. J. Brodsky, et al., Adv. High Energy Phys. 2015 (2015) 231547, 1504.06287.

[19] S. J. Brodsky, P. Hoyer, C. Peterson, and N. Sakai, Phys. Lett. 93B (1980) 451-455.

[20] J. Pumplin, H. L. Lai, and W. K. Tung, Phys. Rev. D75 (2007) 054029, hep-ph/ 0701220. 